\title{
Synthesis and Characterization of Allyl( $\beta$-ketoiminato)palladium(II) Complexes: New Precursors for Chemical Vapor Deposition of Palladium Thin Films
}

\author{
Yeng-Lien Tung, ${ }^{\dagger}$ Wen-Cheng Tseng, ${ }^{\dagger}$ Chi-Y oung Lee, ${ }^{\dagger}$ Peng-F u Hsu, ${ }^{\dagger}$ \\ Yun Chi, ${ }^{*}+{ }^{\dagger}$ Shie-Ming Peng, ${ }^{\ddagger}$ and Gene-Hsiang Lee
}

\begin{abstract}
Department of Chemistry and Material Research Center, National Tsing Hua University, Hsinchu 30043, Republic of China, and Department of Chemistry and Instrumentation Center, National Taiwan University, Taipe 10764, Taiwan, Republic of China
\end{abstract}

Received August 28, 1998

\begin{abstract}
Treatment of $\beta$-ketoiminates with $[(\text { allyl }) \mathrm{Pd}(\mu-\mathrm{Cl})]_{2}$ affords volatile, air-stable allyl $(\beta$ ketoiminato)palladium(II) complexes. The structure of [(methallyl)Pd(Phacac)] (Phacac $=$ $\mathrm{MeCOCHCM} \mathrm{eNPh)} \mathrm{was} \mathrm{determined} \mathrm{by} \mathrm{single-crystal} \mathrm{X-ray} \mathrm{diffraction.} \mathrm{In} \mathrm{solution}{ }^{1} \mathrm{H}$ NMR analyses indicate either a direct rotation of a planar allyl ligand about the allyl-palladium axis or a solvent-assisted exchange process involving the transient formation of a monodentate ketoiminato ligand. The physical properties of the complexes were modified by altering the $\beta$-ketoiminato or the $\eta^{3}$-allyl ligands. Some derivatives are liquid at room temperature and were known to be suitable for chemical vapor deposition of Pd thin films.
\end{abstract}

The preparation of thin films of palladium by metal organic chemical vapor deposition (CVD) is becoming increasingly attractive for the fabrication of electronic devices ${ }^{1}$ because such films have potentially useful applications in replacing gold as electrical contacts in integrated circuits and in making gas sensors, magnetooptical data storage devices, etc. ${ }^{2}$ The largest obstacle to CVD of Pd films has been the lack of suitable precursors, as most volatile Pd complexes suffer from poor stability in air and moisture. ${ }^{3}$ Recently, this problem was partially overcome by Puddephatt, who reported the preparation of vol atile allyl( $\beta$-diketonato)palladium(II) complexes, which are liquid and stable at room temperature and are excellent CVD precursors for $\mathrm{Pd}$ films. ${ }^{4}$ In view of these results, we felt it desirable to investigate analogous complexes having $\beta$-ketoimi-

* To whom correspondence should be addressed. FAX: (886) $3572-$ 0864. E-mail: ychi@chem.nthu.edu.tw.

${ }^{+}$National Tsing Hua University.

₹ National Taiwan University.

(1) (a) Bhaskaran, V.; Hampden-Smith, M.J .; Kodas, T. T. Chem Vap. Deposition 1997, 3, 85. (b) Zhang, Y.; Chio, S. W.-K.; Puddephatt, R. J.J . Am. Chem. Soc. 1997, 119, 9295. (c) Sordelli, L.; Martra, G.; Psaro, R.; Dossi, C.; Coluccia, S. J . Chem. Soc., Dalton Trans. 1996, 765. (d) Thomas, R. R.; Park, J. M. J. Electrochem. Soc. 1989, 136, 1661. (e) Feurer, E.; Suhr, H. Thin Solid Films 1988, 157, 81. (f) Kudo, T.; Yamaguchi, A. J pn. Pat. J P62 207 868, 1987.

(2) (a) Gliem, R.; Schlamp, G. Met. Technol. 1987, 41, 34. (b) Dubin, V. M.; Lopatin, S. D.; Sokolov, V. G. Thin Solid Films 1993, 226, 94. (c) Dossi, C.; Psaro, R.; Bartsch, A.; Brivio, E.; Galasco, A.; Losi, P. Catal. Today 1993, 17, 527. (d) Hierso, J .-C.; Serp, P.; Feurer, R.; Kalck, P. Appl. Organomet. Chem. 1998, 12, 161.

(3) (a) Gozum, J . E.; Poll lina, D. M.; J ensen, J. A.; Girolami, G. S. J Am. Chem. Soc. 1988, 110, 2688. (b) Yuan, Z.; J iang, D.; Naftel, S. J .; Sham, T.-K.; Puddephatt, R. J . Chem. Mater. 1994, 6, 2151.

(4) (a) Yuan, Z.; Puddephatt, R. J . Adv. Mater. 1994, 6, 51. (b) Yuan, Z.; Puddephatt, R. J . Chem. Vap. Deposition 1997, 3, 81. (c) Zang, Y.; Yuan, Z.; Puddephatt, R. J . Chem. Mater. 1998, 10, 2293. nate ligands, which allow for organic functional group variation on the imine nitrogen as a possible way to finetune physical and chemical properties. In this article, we report the synthesis and characterization of such $\beta$-ketoiminato complexes and a preliminary investigation of their application in the generation of Pd films.

\section{Experimental Section}

General Information and Materials. Infrared spectra were recorded on a Perkin-Elmer 2000 FT-IR spectrometer. ${ }^{1} \mathrm{H}$ and ${ }^{13} \mathrm{C}$ NMR spectra were recorded on a Bruker $\mathrm{AM}-400$ or AMX-300 instrument. Mass spectra were obtained on a J EOL SX-102A instrument operating in fast atom bombardment ( $F A B$ ) mode. Thermogravimetric analyses were made with a Seiko TG/DTA 300 instrument. Melting points were determined using Seiko SSC 5000 differential scanning calorimetry. Elemental analyses were performed at the NSC Regional Instrumentation Center at National Cheng Kung University, Tainan, Taiwan.

SEM micrographs of palladium films were obtained using a Hitachi S-4000 scanning electron microscope. Resistivities of films were measured using a LR-400 four-wire AC resistance bridge, manufactured by Linear Research Inc. XPS spectra of films were recorded with a Physical Electronics PHI 1600 ESCA instrument with an $\mathrm{Al} / \mathrm{Mg}$ dual anodeX-ray source. The surface compositions in atomic percentage deduced from XPS spectra were determined after 1-2 min sputtering with argon at $4 \mathrm{keV}$ until a constant composition was obtained. AES spectra for surface scanning and depth profile were recorded on a Physical Electronics PHI $670 \mathrm{Xi}$ Auger instrument after sputtering with argon at $3 \mathrm{keV}$. XPS is most useful for detection of carbon (detection limit, ca. $0.5 \%$ ), while Auger spectra are useful to detect oxygen and fluorine in the presence 
of palladium. In all cases analyses are corrected for relative sensitivities of each element.

Allylpalladium complexes with the empirical formula $[(\text { allyl }) \mathrm{Pd}(u-\mathrm{Cl})]_{2}$ were prepared according to literature procedures. ${ }^{5} \mathrm{~K}$ etoimines were prepared from the direct condensation of acetylacetone and amines at room temperature, ${ }^{6}$ or from reaction of hexafluoroacetylacetone and amines using montmorillonite $\mathrm{K} 10$ as a catalyst. ${ }^{7}$ Abbreviations used for the $\beta$-ketoiminate ligands are as follows: $\mathrm{Mehfac}=\mathrm{CF}_{3} \mathrm{COCHCCF}_{3-}$ $\mathrm{NMe}$, Buhfac $=\mathrm{CF}_{3} \mathrm{COCHCCF}_{3} \mathrm{~N}^{\mathrm{n} B u}, \mathrm{Mohfac}=\mathrm{CF}_{3} \mathrm{COCHC}-$ $\mathrm{CF}_{3} \mathrm{NCH}_{2} \mathrm{CH}_{2} \mathrm{OMe}$, Alhfac $=\mathrm{CF}_{3} \mathrm{COCHCCF}{ }_{3} \mathrm{NCH}_{2} \mathrm{CH}=\mathrm{CH}_{2}$ $\mathrm{Phacac}=\mathrm{MeCOCHCMeNPh}, \mathrm{Meacac}=\mathrm{MeCOCHCMeNMe}$, $\mathrm{M}$ oacac $=\mathrm{MeCOCHCMeNCH}{ }_{2} \mathrm{CH}_{2} \mathrm{OMe}$, Alacac $=\mathrm{MeCOCHC}-$ $\mathrm{MeNCH}{ }_{2} \mathrm{CH}=\mathrm{CH}_{2}$. Two typical synthetic procedures for the preparation of $\beta$-ketoiminato $\mathrm{Pd}$ complexes are described below, together with spectral and analytical data for the compounds prepared. Due to thermal instability or physical state, some compounds did not give satisfactory elemental analyses. ${ }^{1} \mathrm{H}$ NMR spectra for these complexes $(\mathbf{2}, \mathbf{6}, \mathbf{8}-\mathbf{1 1}$, 13, 14) have been deposited in the Supporting Information.

[Pd $\left(\eta^{3}-\mathbf{C H}_{2} \mathbf{C M e C H}_{2}\right)$ (Phacac)] (1). A mixture of distilled water $(40 \mathrm{~mL}), \mathrm{NaOH}(250 \mathrm{mg})$, and PhacacH (175 mg, 1.00 mmol) was stirred at $45^{\circ} \mathrm{C}$ for $30 \mathrm{~min}$ to give a homogeneous solution, which was then added to a suspension of $\left[\mathrm{Pd}\left(\eta^{3}-\mathrm{CH}_{2-}\right.\right.$ $\left.\left.\mathrm{CMeCH}_{2}\right)(\mu-\mathrm{Cl})\right]_{2}(217 \mathrm{mg}, 0.55 \mathrm{mmol})$ in diethyl ether $(20 \mathrm{~mL})$. The mixture was stirred for $10 \mathrm{~min}$, and the ether layer was separated, washed with distilled water $(3 \times 10 \mathrm{~mL})$ and dried over sodium sulfate; then the solvent was evaporated to give the product $\left[\mathrm{Pd}\left(\eta^{3}-\mathrm{CH}_{2} \mathrm{CMeCH}_{2}\right)(\mathrm{Phacac})\right]$ as a yellow solid (yield, 289 mg, 86\%). Single crystals of 1 suitable for an X-ray diffraction study were obtained from recrystallization in saturated acetone solution at room temperature.

[Pd $\left(\eta^{3}-\mathrm{CH}_{2} \mathbf{C M e C H}_{2}\right)$ (Mehfac)] (15). A mixture of di ethyl ether (30 mL), NaOMe (200 mg), and MehfacH (450 mg, 2.04 $\mathrm{mmol}$ ) was stirred at room temperature for $30 \mathrm{~min}$ to give a homogeneous solution, which was then added to a mixture of $\left[\mathrm{Pd}\left(\eta^{3}-\mathrm{CH}_{2} \mathrm{CMeCH}_{2}\right)(\mu-\mathrm{Cl})\right]_{2}(400 \mathrm{mg}, 1.02 \mathrm{mmol})$ and $\mathrm{NaOH}$ $(250 \mathrm{mg}$ ) in water $(50 \mathrm{~mL})$. The mixture was vigorously stirred for $10 \mathrm{~min}$; the ether layer was separated, washed with distilled water $(3 \times 10 \mathrm{~mL})$, and dried over sodium sulfate. Then the solvent was evaporated to give the product $\left[\mathrm{Pd}\left(\eta^{3}-\right.\right.$ $\mathrm{CH}_{2} \mathrm{CMeCH}_{2}$ )(Mehfac)] as a yell ow solid (yield, $740 \mathrm{mg}, 95 \%$ ).

Spectroscopic Data for $\beta$-Ketoiminato Complexes. Complex 1, [Pd $\left(\eta^{3}-\mathrm{CH}_{\mathbf{2}} \mathbf{C M e C H _ { 2 } ) ( P h a c a c ) ] . ~ M S ~ ( F A B , ~}\right.$ $\left.{ }^{107} \mathrm{Pd}\right): \mathrm{m} / \mathrm{z} 335\left(\mathrm{M}^{+}\right) .{ }^{1} \mathrm{H}$ NMR $\left(300 \mathrm{MHz}, \mathrm{CDCl}_{3}, 293 \mathrm{~K}\right): \delta$ $7.24(\mathrm{t}, 2 \mathrm{H}, \mathrm{J} \mathrm{нн}=7.5 \mathrm{~Hz}), 7.00(\mathrm{t}, 1 \mathrm{H}, \mathrm{J} \mathrm{HH}=7.5 \mathrm{~Hz}), 6.88(\mathrm{br}$, $1 \mathrm{H}), 4.97(\mathrm{~s}, 1 \mathrm{H}), 3.52(\mathrm{~d}, 1 \mathrm{H}, \mathrm{J} \mathrm{нн}=2.8 \mathrm{~Hz}), 2.73(\mathrm{~s}, 1 \mathrm{H}), 2.34$ $(\mathrm{s}, 1 \mathrm{H}), 2.03(\mathrm{~s}, 3 \mathrm{H}), 1.98(\mathrm{~s}, 3 \mathrm{H}), 1.92(\mathrm{~d}, 1 \mathrm{H}, \mathrm{J} \mathrm{Hн}=2.8 \mathrm{~Hz})$,

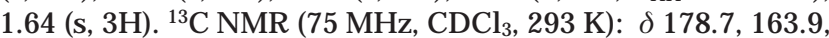
156.9, 130.5, 128.5 (2C), 123.7 (2C), 122.8, 97.3, 58.8, 57.1, 27.1, 23.3, 22.9. Anal. Calcd for $\mathrm{C}_{15} \mathrm{H}_{19} \mathrm{NOPd}$ : C, 53.67; $\mathrm{H}, 5.70 ; \mathrm{N}$, 4.17. Found: $C, 53.19 ; \mathrm{H}, 5.65 ; \mathrm{N}, 4.30$.

Complex 2, [Pd $\left(\eta^{3}-\mathbf{C H}_{\mathbf{2}} \mathbf{C H C H}\right.$ )(Meacac)]. ${ }^{1} \mathrm{H}$ NMR (300 $\left.\mathrm{MHz} \mathrm{CDCl}_{3}, 293 \mathrm{~K}\right): \delta 5.67-5.54(\mathrm{~m}, 1 \mathrm{H}), 4.80(\mathrm{~s}, 1 \mathrm{H}), 3.78$ $(\mathrm{d}, 1 \mathrm{H}, \mathrm{J}$ нн $=6.9 \mathrm{~Hz}), 3.49(\mathrm{~s}, 3 \mathrm{H}), 3.22(\mathrm{~d}, 1 \mathrm{H}, \mathrm{J} \mathrm{HH}=6.6 \mathrm{~Hz})$, $3.01\left(\mathrm{~d}, 1 \mathrm{H}, \mathrm{J}_{\text {нн }}=12.5 \mathrm{~Hz}\right), 2.71\left(\mathrm{~d}, 1 \mathrm{H}, \mathrm{J}_{\mathrm{HH}}=11.7 \mathrm{~Hz}\right), 2.00$ (s, 3H), $1.95(\mathrm{~s}, 3 \mathrm{H}) \cdot{ }^{13} \mathrm{C}$ NMR $\left(75 \mathrm{MHz}, \mathrm{CDCl}_{3}, 293 \mathrm{~K}\right): \delta$ 175.8, 165.4, 115.1, 97.8, 60.0, 53.2, 49.1, 26.3, 21.5.

Complex 3, [Pd $\left(\eta^{3}-\mathrm{CH}_{2} \mathrm{CHCHMe}\right)($ Meacac)]. MS (FAB, $\left.{ }^{107} \mathrm{Pd}\right): \mathrm{m} / \mathrm{z} 273\left(\mathrm{M}^{+}\right) .{ }^{1} \mathrm{H}$ NMR $\left(300 \mathrm{MHz} \mathrm{CDCl}_{3}, 293 \mathrm{~K}\right): \delta$ $4.78(\mathrm{~s}, 1 \mathrm{H}), 3.54\left(\mathrm{~d}, 1 \mathrm{H}, \mathrm{J}_{\text {нн }}=2.6 \mathrm{~Hz}\right), 3.45(\mathrm{~s}, 3 \mathrm{H}), 2.96(\mathrm{~d}$, $1 \mathrm{H}, \mathrm{J} \mathrm{нH}=2.6 \mathrm{~Hz}), 2.84(\mathrm{~s}, 1 \mathrm{H}), 2.62(\mathrm{~s}, 1 \mathrm{H}), 2.10(\mathrm{~s}, 3 \mathrm{H}), 1.91$ (s, 3H), $1.86(\mathrm{~s}, 3 \mathrm{H}) .{ }^{13} \mathrm{C}$ NMR $\left(75 \mathrm{MHz} \mathrm{CDCl}_{3}, 293 \mathrm{~K}\right): \delta$ $176.1,165.4,130.7,97.7,58.5,53.5,49.1,26.5,23.4,21.5$. Anal.

(5) (a) Robinson, S. D.; Shaw, B. L. J . Chem. Soc. 1963, 4806. (b) Sakakibara, M.; Takahashi, Y.; Sakai, S.; I shii, Y.J. Chem. Soc., Chem. Commun. 1969, 396. (c) Akermark, B.; Hansson, S.; Krakenberger, B.; Vitagliano, A.; Zetterberg, K. Organometallics 1984, 3, 679.

(6) Greenhill, J . V. J . Chem. Soc. Rev. 1977, 6, 277.

(7) Braibante, M. E. F.; Braibante, H. S.; Missio, L.; Andricopulo, A. Synthesis 1994, 898.
Calcd for $\mathrm{C}_{15} \mathrm{H}_{19} \mathrm{NOPd:} \mathrm{C}, 43.89 ; \mathrm{H}, 6.28 ; \mathrm{N}, 5.12$. Found: $\mathrm{C}$, 44.27; H, 6.21; N, 5.28.

Complex 4, [Pd( $\left.\eta^{3}-\mathrm{CH}_{\mathbf{2}} \mathrm{CMeCH}_{\mathbf{2}}\right)$ (Meacac)]. MS (FAB, $\left.{ }^{107} \mathrm{Pd}\right): \mathrm{m} / \mathrm{z} 273\left(\mathrm{M}^{+}\right) .{ }^{1} \mathrm{H} \mathrm{NMR}\left(300 \mathrm{MHz} \mathrm{CDCl}_{3}, 293 \mathrm{~K}\right): \delta$ 5.31-5.21 (m, $1 \mathrm{H}), 4.74(\mathrm{~s}, 1 \mathrm{H}), 3.70-3.60(\mathrm{~m}, 1 \mathrm{H}), 3.45(\mathrm{~s}$, $3 \mathrm{H}), 3.00(\mathrm{~d}, 1 \mathrm{H}, \mathrm{J} \mathrm{HH}=6.7 \mathrm{~Hz}), 2.46\left(\mathrm{~d}, 1 \mathrm{H}, \mathrm{J} \mathrm{HH}^{2}=11.5 \mathrm{~Hz}\right)$, $1.94(\mathrm{~s}, 3 \mathrm{H}), 1.90(\mathrm{~s}, 3 \mathrm{H}), 1.29(\mathrm{~d}, 3 \mathrm{H}, \mathrm{J}$ нн $=6.3 \mathrm{~Hz}) .{ }^{13} \mathrm{C} \mathrm{NMR}$ $\left(75 \mathrm{MHz}_{1} \mathrm{CDCl}_{3}, 293 \mathrm{~K}\right.$ ): $\delta 177.1,165.4,114.3,97.7,75.6,49.3$, 49.1, 26.9, 21.2, 16.1. Anal. Calcd for $\mathrm{C}_{10} \mathrm{H}_{17}$ NOPd: C, 43.89; $\mathrm{H}, 6.26$; N, 5.12. Found: C, 44.20; H, 6.12; N, 5.22.

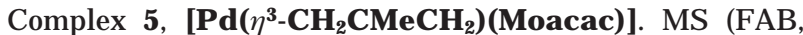
$\left.{ }^{107} \mathrm{Pd}\right): \mathrm{m} / \mathrm{z} 317\left(\mathrm{M}^{+}\right) .{ }^{1} \mathrm{H}$ NMR $\left(300 \mathrm{MHz}, \mathrm{CDCl}_{3}, 293 \mathrm{~K}\right): \delta$ $5.31-5.21(\mathrm{~m}, 1 \mathrm{H}), 4.93(\mathrm{~s}, 1 \mathrm{H}), 3.96-3.87(\mathrm{~m}, 1 \mathrm{H}), 3.85-3.76$ $(\mathrm{m}, 1 \mathrm{H}), 3.71-3.61(\mathrm{~m}, 1 \mathrm{H}), 3.50(\mathrm{t}, 2 \mathrm{H}, \mathrm{J} \mathrm{HH}=6.6 \mathrm{~Hz}), 3.29$ $(\mathrm{s}, 3 \mathrm{H}), 2.93(\mathrm{~d}, 1 \mathrm{H}, \mathrm{J} \mathrm{HH}=6.5 \mathrm{~Hz}), 2.44(\mathrm{~d}, 1 \mathrm{H}, \mathrm{J} \mathrm{HH}=11.6$ $\mathrm{Hz}), 1.93(\mathrm{~s}, 3 \mathrm{H}), 1.91(\mathrm{~s}, 3 \mathrm{H}), 1.31(\mathrm{~d}, 3 \mathrm{H}, \mathrm{J} \mathrm{нH}=6.2 \mathrm{~Hz}) \cdot{ }^{13} \mathrm{C}$ NMR $\left(75 \mathrm{MHz} \mathrm{CDCl}_{3}, 293 \mathrm{~K}\right): \delta 177.8,165.3,133.7,98.1$, 76.2, 73.5, 59.3, 59.0, 48.8, 27.1, 21.2, 16.0. Anal. Calcd for $\mathrm{C}_{12} \mathrm{H}_{21} \mathrm{NO}_{2} \mathrm{Pd}$ : C, 45.37; $\mathrm{H}, 6.66 ; \mathrm{N}, 4.41$. Found: $\mathrm{C}, 45.80 ; \mathrm{H}$, 6.56; N, 4.60 .

Complex 6, [Pd $\left(\eta^{3}-\mathrm{CH}_{2} \mathrm{CMeCH}_{2}\right)($ Alacac) ]: MS (FAB, $\left.{ }^{107} \mathrm{Pd}\right): \mathrm{m} / \mathrm{z} 299\left(\mathrm{M}^{+}\right) .{ }^{1} \mathrm{H} \mathrm{NMR}\left(300 \mathrm{MHz} \mathrm{CDCl}_{3}, 293 \mathrm{~K}\right): \delta$ $5.91-5.79(\mathrm{~m}, 1 \mathrm{H}), 5.14-5.07(\mathrm{~m}, 2 \mathrm{H}), 4.80(\mathrm{~s}, 1 \mathrm{H}), 4.36-4.33$ $(\mathrm{m}, 2 \mathrm{H}), 3.56(\mathrm{~d}, 1 \mathrm{H}, \mathrm{J}$ нн $=2.8 \mathrm{~Hz}), 2.86\left(\mathrm{~d}, 1 \mathrm{H}, \mathrm{J}_{\mathrm{HH}}=2.8\right.$ $\mathrm{Hz}), 2.82(\mathrm{~s}, 1 \mathrm{H}), 2.60(\mathrm{~s}, 1 \mathrm{H}), 2.08(\mathrm{~s}, 3 \mathrm{H}), 1.96(\mathrm{~s}, 3 \mathrm{H}), 1.88$ (s, 3H). ${ }^{13} \mathrm{C} \mathrm{NMR}\left(75 \mathrm{MHz}, \mathrm{CDCl}_{3}, 293 \mathrm{~K}\right): \delta 176.8,165.5$, 135.6, 130.6, 114.6, 98.0, 62.0, 58.4, 54.5, 26.7, 23.2, 21.0.

Complex 7, [Pd( $\left.\eta^{3}-\mathrm{CH}_{2} \mathrm{CMeCHMe}\right)($ Meacac) ]: MS (FAB, $\left.{ }^{107} \mathrm{Pd}\right): \mathrm{m} / \mathrm{z} 287\left(\mathrm{M}^{+}\right) .{ }^{1} \mathrm{H} N M R\left(300 \mathrm{MHz}, \mathrm{CDCl}_{3}, 293 \mathrm{~K}\right): \delta$ $5.06(\mathrm{dd}, 1 \mathrm{H}, \mathrm{J} \mathrm{нн}=12.0,7.1 \mathrm{~Hz}), 4.72(\mathrm{~s}, 1 \mathrm{H}), 3.45(\mathrm{~s}, 3 \mathrm{H})$, $3.00\left(\mathrm{~d}, 1 \mathrm{H}, \mathrm{J}_{\mathrm{HH}}=7.1 \mathrm{~Hz}\right), 2.64\left(\mathrm{~d}, 1 \mathrm{H}, \mathrm{J}_{\mathrm{HH}}=12.0 \mathrm{~Hz}\right), 1.89$ $(\mathrm{s}, 3 \mathrm{H}), 1.84(\mathrm{~s}, 3 \mathrm{H}), 1.40(\mathrm{~s}, 3 \mathrm{H}), 1.20(\mathrm{~s}, 3 \mathrm{H}) .{ }^{13} \mathrm{C}$ NMR $(75$ $\left.\mathrm{MHz}_{2} \mathrm{CDCl}_{3}, 293 \mathrm{~K}\right): \delta 177.8,165.6,108.5,97.5,88.7,49.1$, 46.7, 27.2, 25.0, 21.0, 20.9. Anal. Calcd for $\mathrm{C}_{11} \mathrm{H}_{19} \mathrm{NOPd:} \mathrm{C}_{\text {, }}$ 45.93; H, 6.66; N, 4.87. Found: C, 46.66; H, 6.46; N, 4.95.

Complex 8, [Pd( $\left.\eta^{3}-\mathrm{CH}_{2} \mathrm{CHCMe}_{2}\right)$ (Moacac)]. MS (FAB, $\left.{ }^{107} \mathrm{Pd}\right): \mathrm{m} / \mathrm{z} 331\left(\mathrm{M}^{+}\right) .{ }^{1} \mathrm{H}$ NMR $\left(300 \mathrm{MHz} \mathrm{CDCl}_{3}, 293 \mathrm{~K}\right): \delta$ $5.09(\mathrm{dd}, 1 \mathrm{H}, \mathrm{J}$ нн $=12.0 \mathrm{~Hz}, 7.2 \mathrm{~Hz}), 4.74(\mathrm{~s}, 1 \mathrm{H}), 3.97-3.80$ $(\mathrm{m}, 2 \mathrm{H}), 3.54(\mathrm{t}, 2 \mathrm{H}, \mathrm{J}$ нн $=3 \mathrm{~Hz}), 3.33(\mathrm{~s}, 3 \mathrm{H}), 2.96(\mathrm{dd}, 1 \mathrm{H}$, J нн $=7.3 \mathrm{~Hz}, 1.3 \mathrm{~Hz}), 2.63(\mathrm{dd}, 1 \mathrm{H}, \mathrm{J} \mathrm{нн}=12.0,1.3 \mathrm{~Hz}), 1.93$ $(\mathrm{s}, 3 \mathrm{H}), 1.92(\mathrm{~s}, 3 \mathrm{H}), 1.44(\mathrm{~s}, 3 \mathrm{H}), 1.22(\mathrm{~s}, 3 \mathrm{H}) .{ }^{13} \mathrm{C}$ NMR $(75$ $\left.\mathrm{MHz} \mathrm{CDCl}_{3}, 293 \mathrm{~K}\right): \delta 178.6,165.5,108.1,98.1,86.6,73.5$, 59.3, 59.1, 46.2, 27.4, 25.1, 21.1, 21.0.

Complex 9, [Pd $\left.\left(\eta^{3}-\mathrm{CH}_{2} \mathrm{CMeCMeCH}{ }_{2} \mathrm{OMe}\right)(\mathrm{Moacac})\right]$ : MS (FAB, $\left.{ }^{107} \mathrm{Pd}\right): \mathrm{m} / \mathrm{z} 375\left(\mathrm{M}^{+}\right)$. ${ }^{1} \mathrm{H}$ NMR $\left(300 \mathrm{MHz}, \mathrm{CDCl}_{3}, 293\right.$ $\mathrm{K}): \delta 4.74(\mathrm{~s}, 1 \mathrm{H}), 3.87(\mathrm{t}, 2 \mathrm{H}, \mathrm{J} \mathrm{нн}=6.3 \mathrm{~Hz}), 3.64(\mathrm{~d}, 1 \mathrm{H}, \mathrm{J} \mathrm{нH}$ $=10.6 \mathrm{~Hz}), 3.51(\mathrm{t}, 2 \mathrm{H}, \mathrm{J}$ нн $=6.3 \mathrm{~Hz}), 3.32(\mathrm{~s}, 3 \mathrm{H}), 3.16(\mathrm{~s}$, $3 \mathrm{H}), 3.10(\mathrm{~d}, 1 \mathrm{H}, \mathrm{J} \mathrm{нH}=10.6 \mathrm{~Hz}), 2.96(\mathrm{~d}, 1 \mathrm{H}, \mathrm{J} \mathrm{нH}=1.1 \mathrm{~Hz})$, $2.86(\mathrm{~d}, 1 \mathrm{H}, \mathrm{J} \mathrm{HH}=1.1 \mathrm{~Hz}), 2.11(\mathrm{~s}, 3 \mathrm{H}), 1.92(\mathrm{~s}, 3 \mathrm{H}), 1.91(\mathrm{~s}$, $3 \mathrm{H}), 1.40(\mathrm{~s}, 3 \mathrm{H}) .{ }^{13} \mathrm{C}$ NMR $\left(75 \mathrm{MHz} \mathrm{CDCl}_{3}, 293 \mathrm{~K}\right): \delta 178.3$, $165.4,123.5,98.0,80.7,73.4,73.0,59.1$ (2C), 57.3, 51.6, 27.2, $21.2(2 \mathrm{C}), 17.1$

Complex 10, [Pd $\left(\eta^{3}-\mathrm{CH}_{2} \mathrm{CMeCMeCH} \mathrm{OMMe}_{2}\right.$ (Mehfac)] MS (FAB, $\left.{ }^{107} \mathrm{Pd}\right): \mathrm{m} / \mathrm{z} 439\left(\mathrm{M}^{+}\right)$. ${ }^{1} \mathrm{H}$ NMR $\left(300 \mathrm{MHz} \mathrm{CDCl}_{3}\right.$ $293 \mathrm{~K}): \delta 5.60(\mathrm{~s}, 1 \mathrm{H}), 3.74(\mathrm{~d}, 3 \mathrm{H}, \mathrm{J} \mathrm{HH}=2.0 \mathrm{~Hz}), 3.62(\mathrm{~d}, 1 \mathrm{H}$, J нн $=10.7 \mathrm{~Hz}), 3.32(\mathrm{~s}, 1 \mathrm{H}), 3.18(\mathrm{~s}, 3 \mathrm{H}), 3.12(\mathrm{~d}, 1 \mathrm{H}, \mathrm{J} \mathrm{нH}=$ $10.7 \mathrm{~Hz}), 3.11(\mathrm{~s}, 1 \mathrm{H}), 2.17(\mathrm{~s}, 3 \mathrm{H}), 1.39(\mathrm{~s}, 3 \mathrm{H}) .{ }^{13} \mathrm{C}$ NMR $(75$ $\left.\mathrm{MHz} \mathrm{CDCl}_{3}, 293 \mathrm{~K}\right): \delta 166.1\left(\mathrm{q}, \mathrm{CO}, \mathrm{J}_{\mathrm{CF}}=32 \mathrm{~Hz}\right), 155.6(\mathrm{q}$, $\mathrm{CN}$, J $\mathrm{CF}=26 \mathrm{~Hz}), 126.9,120.1\left(\mathrm{q}, \mathrm{CF}_{3}\right.$, J $\left.\mathrm{CF}=282 \mathrm{~Hz}\right), 120.0$ $\left(q, \mathrm{CF}_{3}\right.$, J $\left.\mathrm{CF}=286 \mathrm{~Hz}\right), 87.5,84.9,73.7,58.3,55.1,50.7,21.6$, 18.2 .

Complex 11, [Pd( $\left.\eta^{3}-\mathrm{CH}_{2} \mathrm{CMeCMeCH}{ }_{2} \mathrm{OMe}\right)($ Mohfac)] ${ }^{1} \mathrm{H}$ NMR $\left(300 \mathrm{MHz} \mathrm{CDCl}_{3}, 293 \mathrm{~K}\right): \delta 5.61(\mathrm{~s}, 1 \mathrm{H}), 4.10-4.03$ $(\mathrm{m}, 2 \mathrm{H}, \mathrm{J} \mathrm{нн}=6.9 \mathrm{~Hz}), 3.65-3.57(\mathrm{~m}, 3 \mathrm{H}), 3.38(\mathrm{~d}, 1 \mathrm{H}, \mathrm{J} \mathrm{нн}=$ $10.6 \mathrm{~Hz}), 3.35$ (s, 3H), $3.19(\mathrm{~s}, 3 \mathrm{H}), 3.13(\mathrm{~s}, 1 \mathrm{H}), 3.10(\mathrm{~d}, 1 \mathrm{H}$, $\mathrm{J} \mathrm{HH}=10.6 \mathrm{~Hz}), 2.19(\mathrm{~s}, 3 \mathrm{H}), 1.40(\mathrm{~s}, 3 \mathrm{H}) .{ }^{13} \mathrm{C} N M R(75 \mathrm{MHz}$, $\left.\mathrm{CDCl}_{3}, 293 \mathrm{~K}\right): \delta 166.4\left(\mathrm{q}, \mathrm{CO}, \mathrm{J}_{\mathrm{CF}}=32 \mathrm{~Hz}\right), 156.1(\mathrm{q}, \mathrm{CN}$, $\mathrm{J}_{\mathrm{CF}}=26 \mathrm{~Hz}$ ), 126.6, $120.0\left(\mathrm{q}, \mathrm{CF}_{3}, \mathrm{~J}_{\mathrm{CF}}=286 \mathrm{~Hz}\right), 119.9(\mathrm{q}$, $\left.\mathrm{CF}_{3}, \mathrm{~J}_{\mathrm{CF}}=279 \mathrm{~Hz}\right), 87.6,85.2,74.6,73.6,60.8,59.8,58.4,55.6$, 21.5, 18.2. 
Complex 12, [Pd( $\left.\left.\eta^{3}-\mathrm{CH}_{\mathbf{2}} \mathrm{CHCH}_{\mathbf{2}}\right)(\mathrm{Mehfac})\right]$. MS (FAB, $\left.{ }^{107} \mathrm{Pd}\right): \mathrm{m} / \mathrm{z} 367\left(\mathrm{M}^{+}\right) .{ }^{1} \mathrm{H} \mathrm{NMR}\left(300 \mathrm{MHz} \mathrm{CDCl}_{3}, 293 \mathrm{~K}\right): \delta$ $5.82-5.67(\mathrm{~m}, 1 \mathrm{H}), 5.67(\mathrm{~s}, 1 \mathrm{H}), 4.07(\mathrm{~d}, 1 \mathrm{H}, \mathrm{J} \mathrm{HH}=6.8 \mathrm{~Hz})$, $3.78(\mathrm{~s}, 3 \mathrm{H}), 3.53(\mathrm{~d}, 1 \mathrm{H}$, J нн $=6.5 \mathrm{~Hz}), 3.24(\mathrm{~d}, 1 \mathrm{H}, \mathrm{J} \mathrm{нн}=$ $12.4 \mathrm{~Hz}), 2.94(\mathrm{~d}, 1 \mathrm{H}, \mathrm{J} \mathrm{нн}=11.8 \mathrm{~Hz}) .{ }^{13} \mathrm{C} \mathrm{NMR}(75 \mathrm{MHz}$, $\left.\mathrm{CDCl}_{3}, 293 \mathrm{~K}\right): \delta 165.6\left(\mathrm{q}, \mathrm{CO}, \mathrm{J}_{\mathrm{CF}}=33 \mathrm{~Hz}\right), 155.6(\mathrm{q}, \mathrm{CN}$, $\mathrm{J} \mathrm{CF}=27 \mathrm{~Hz}$ ), $120.2\left(\mathrm{q}, \mathrm{CF}_{3}\right.$ J J $\left.\mathrm{CF}=282 \mathrm{~Hz}\right), 120.0\left(\mathrm{q}, \mathrm{CF}_{3}\right.$, J $\mathrm{CF}$ $=286 \mathrm{~Hz}), 117.8,87.5,63.3,57.1,50.9$. Anal. Calcd for $\mathrm{C}_{9} \mathrm{H}_{9}-$ NOF ${ }_{6} \mathrm{Pd}:$ C, 29.41; H, 2.47; N, 3.81. Found: C, 30.30; H, 2.64; N, 4.05.

Complex 13, [Pd( $\left.\left.\eta^{3}-\mathrm{CH}_{\mathbf{2}} \mathbf{C H C H}\right)(B u h f a c)\right] .{ }^{1} \mathrm{H}$ NMR (300 $\left.\mathrm{MHz} \mathrm{CDCl}_{3}, 293 \mathrm{~K}\right): \delta 5.81-5.68(\mathrm{~m}, 1 \mathrm{H}), 5.64(\mathrm{~s}, 1 \mathrm{H}), 4.07$ $(\mathrm{d}, 1 \mathrm{H}, \mathrm{J} \mathrm{HH}=6.7 \mathrm{~Hz}), 3.89-3.79(\mathrm{~m}, 2 \mathrm{H}), 3.41\left(\mathrm{~d}, 1 \mathrm{H}, \mathrm{J} \mathrm{HH}_{\mathrm{H}}=\right.$ $6.7 \mathrm{~Hz}), 3.22(\mathrm{~d}, 1 \mathrm{H}, \mathrm{J} \mathrm{HH}=12.2 \mathrm{~Hz}), 2.94(\mathrm{~d}, 1 \mathrm{H}, \mathrm{J} \mathrm{HH}=12.2$ $\mathrm{Hz}), 1.71(\mathrm{~m}, 2 \mathrm{H}, \mathrm{J}$ нн $=7.2 \mathrm{~Hz}), 1.34\left(\mathrm{~m}, 2 \mathrm{H}, \mathrm{J} \mathrm{нн}^{2}=7.2 \mathrm{~Hz}\right)$, 0.93 (t, 3H, J нH $=7.2 \mathrm{~Hz}) .{ }^{13} \mathrm{C} \mathrm{NMR}\left(75 \mathrm{MHz}, \mathrm{CDCl}_{3}, 293 \mathrm{~K}\right)$ : $\delta 165.6\left(\mathrm{q}, \mathrm{CO}, \mathrm{J}_{\mathrm{CF}}=32 \mathrm{~Hz}\right), 154.5\left(\mathrm{q}, \mathrm{CN}, \mathrm{J}_{\mathrm{CF}}=26 \mathrm{~Hz}\right), 120.2$ $\left(q, \mathrm{CF}_{3}, J_{\mathrm{CF}}=277 \mathrm{~Hz}\right), 120.1\left(\mathrm{q}, \mathrm{CF}_{3}, J_{\mathrm{CF}}=286 \mathrm{~Hz}\right), 117.1$, 87.4, 63.4, 62.3, 56.6, 36.5, 20.9, 14.5 .

Complex 14, [Pd $\left(\eta^{3}-\mathrm{CH}_{2} \mathrm{CHCH}_{2}\right)$ (Mohfac)]. MS (FAB, $\left.{ }^{107} \mathrm{Pd}\right): \mathrm{m} / \mathrm{z} 411\left(\mathrm{M}^{+}\right) .{ }^{1} \mathrm{H} \mathrm{NMR}\left(300 \mathrm{MHz} \mathrm{CDCl}_{3}, 293 \mathrm{~K}\right): \delta$ $5.82-5.68(\mathrm{~m}, 1 \mathrm{H}), 5.66(\mathrm{~s}, 1 \mathrm{H}), 4.08\left(\mathrm{~m}, 2 \mathrm{H}, \mathrm{J} \mathrm{нн}^{2}=6.7 \mathrm{~Hz}\right)$, 4.09-4.06 (m, 1H), $3.61\left(\mathrm{t}, 1 \mathrm{H}, \mathrm{J}_{\text {нн }}=6.7 \mathrm{~Hz}\right), 3.53(\mathrm{~d}, 1 \mathrm{H}$, $\left.\mathrm{J}_{н H}=6.1 \mathrm{~Hz}\right), 3.33(\mathrm{~s}, 3 \mathrm{H}), 3.21\left(\mathrm{~d}, 1 \mathrm{H}, \mathrm{J}_{н H}=12.4 \mathrm{~Hz}\right), 2.95$ $\left(\mathrm{d}, 1 \mathrm{H}, \mathrm{J}_{\mathrm{Hн}}=11.9 \mathrm{~Hz}\right) .{ }^{13} \mathrm{C} \mathrm{NMR}\left(75 \mathrm{MHz}, \mathrm{CDCl}_{3}, 293 \mathrm{~K}\right): \delta$ 166.3 (q, CO, J CF $=34 \mathrm{~Hz}$ ), 156.1 (q, CN, J $\mathrm{CF}=27 \mathrm{~Hz}), 120.0$ $\left(q, 2 \mathrm{CF}_{3}, \mathrm{~J}_{\mathrm{CF}}=287 \mathrm{~Hz}\right), 117.3,87.6,74.4,63.4,61.1,59.8,57.4$. Anal. Calcd for $\mathrm{C}_{11} \mathrm{H}_{13} \mathrm{NO}_{2} \mathrm{~F}_{6} \mathrm{Pd}$ : C, 32.09; $\mathrm{H}, 3.18 ; \mathrm{N}, 3.40$. Found: C, 30.89; $\mathrm{H}, 3.07$; N, 3.30.

Complex 15, [Pd( $\left.\eta^{3}-\mathrm{CH}_{2} \mathrm{CMeCH}_{2}\right)$ (Mehfac)]. MS (FAB, $\left.{ }^{107} \mathrm{Pd}\right): \mathrm{m} / \mathrm{z} 381\left(\mathrm{M}^{+}\right) .{ }^{1} \mathrm{H}$ NMR $\left(300 \mathrm{MHz} \mathrm{CDCl}_{3}, 293 \mathrm{~K}\right): \delta$ 5.65 (s, $1 \mathrm{H}$ ), $3.84(\mathrm{~d}, 1 \mathrm{H}, \mathrm{J} \mathrm{HH}=2.2 \mathrm{~Hz}), 3.77$ (q, 3H, J HF $=2.0$ $\mathrm{Hz}), 3.30(\mathrm{~d}, 1 \mathrm{H}, \mathrm{J}$ нн $=2.2 \mathrm{~Hz}$ ), $3.08(\mathrm{~s}, 1 \mathrm{H}), 2.84(\mathrm{~s}, 1 \mathrm{H}), 2.19$ (s, 3H). ${ }^{13} \mathrm{C}$ NMR $\left(75 \mathrm{MHz} \mathrm{CDCl}_{3}, 293 \mathrm{~K}\right): \delta 165.5$ (q, CO, $J_{C F}=32 \mathrm{~Hz}$ ), $155.4\left(q, C N, J_{C F}=26 \mathrm{~Hz}\right), 134.0,120.2$ (q, $\mathrm{CF}_{3}$, $\left.J_{C F}=282 \mathrm{~Hz}\right), 120.0\left(q, C_{3}, J_{C F}=285 \mathrm{~Hz}\right), 87.4,61.7,57.0$, 50.8, 23.9. Anal. Calcd for $\mathrm{C}_{10} \mathrm{H}_{11} \mathrm{NOF}_{6} \mathrm{Pd}$ : C, 31.47; $\mathrm{H}, 2.91$; N, 3.67. Found: C, 32.15; H, 2.90; N, 3.88.

Complex 16, [Pd $\left.\left(\eta^{3}-\mathrm{CH}_{2} \mathrm{CMeCH}_{2}\right)(\mathrm{Alhfac})\right]$. MS (FAB, ${ }^{107} \mathrm{Pd}: \mathrm{m} / \mathrm{z} 407\left(\mathrm{M}^{+}\right) .{ }^{1} \mathrm{H}$ NMR $\left(300 \mathrm{MHz}, \mathrm{CDCl}_{3}, 293 \mathrm{~K}\right): \delta$ $5.96-5.84(\mathrm{~m}, 1 \mathrm{H}), 5.68(\mathrm{~s}, 1 \mathrm{H}), 5.20(\mathrm{~d}, 1 \mathrm{H}, \mathrm{J} \mathrm{HH}=10.2 \mathrm{~Hz})$, $5.12(\mathrm{~d}, 1 \mathrm{H}, \mathrm{J} \mathrm{HH}=18.0 \mathrm{~Hz}), 4.57(\mathrm{~s}, 2 \mathrm{H}), 3.84\left(\mathrm{~d}, 1 \mathrm{H}, \mathrm{J} \mathrm{HH}^{=}\right.$ $2.7 \mathrm{~Hz}), 3.18(\mathrm{~d}, 1 \mathrm{H}, \mathrm{J}$ нH $=2.7 \mathrm{~Hz}), 3.18(\mathrm{~s}, 1 \mathrm{H}), 2.82(\mathrm{~s}, 1 \mathrm{H})$, $2.12(\mathrm{~s}, 3 \mathrm{H}) .{ }^{13} \mathrm{C} N M R\left(75 \mathrm{MHz} \mathrm{CDCl}_{3}, 293 \mathrm{~K}\right): \delta 166.2(\mathrm{q}$, $\left.\mathrm{CO}, \mathrm{J}_{\mathrm{CF}}=32 \mathrm{~Hz}\right), 155.4\left(\mathrm{q}, \mathrm{CN}, \mathrm{J}_{\mathrm{CF}}=26 \mathrm{~Hz}\right), 136.4,133.6$, $120.2\left(q, C F_{3}, J_{C F}=282 \mathrm{~Hz}\right), 120.1\left(q, C_{3}, J_{C F}=286 \mathrm{~Hz}\right)$, 116.6, 87.3, 66.5, 63.4, 58.3, 23.5. Anal. Cal cd for $\mathrm{C}_{12} \mathrm{H}_{13} \mathrm{NOF}_{6-}$ Pd: C, 35.36; H, 3.21; N, 3.44. Found: C, 35.97; H, 3.14; N, 3.61.

X-ray Crystallography. The X-ray diffraction measurement was carried out on a Nonius CAD-4 diffractometer at room temperature. Lattice parameters were determined from 25 randomly selected high-angle reflections. Three standard reflections were monitored every 3600 s. No significant variation in intensities $(\leq 1 \%)$ was observed during the course of all data collection. Intensities of diffraction signals were corrected for Lorentz, polarization, and absorption effects ( $\psi$ scans). The structure was solved with the NRCC-SDP-VAX package. All non-hydrogen atoms had anisotropic temperature factors, while hydrogen atoms of organic substituents were placed at calculated positions with $U_{H}=U_{C}+0.1$. The crystallographic refinement parameters of complex $\mathbf{1}$ are summarized in Table 1.

CVD Procedures. Thermal CVD reactions were conducted in a horizontal hot-wall Pyrex reactor, consisting of a Pyrex tube of internal diameter $25 \mathrm{~mm}$, placed within an electric temperature-controlled tube furnace, as described in Chart 1. Precursors were loaded in the glass container. The carrier gas was introduced through a side arm of the container and became saturated with vapors of palladium precursors before entering the hot zone. Si substrates were cleaned using the

\section{Table 1. Crystal Data for the X-ray Diffraction} Studies of Complex 1

formula: $\mathrm{C}_{15} \mathrm{H}_{19} \mathrm{NOPd}$

cryst syst: orthorhombic

mol wt: 335.72

space group: $\mathrm{Pbca}$

$\mathrm{a}=9.0500(20) \AA$

$\mathrm{b}=19.2745(20) \AA$

$\mathrm{c}=16.681(14) \AA$

$V=2910(3) \AA^{3}$

$\mathrm{Z}=8$

$\mathrm{D}_{\mathrm{c}}=1.533 \mathrm{~g} / \mathrm{cm}^{3}$

$\mathrm{F}(000)=1351$

$2 \theta(\max )=50^{\circ}$

h, k, I ranges: $0-10,0-22,0-19$

cryst size: $0.50 \times 0.45 \times 0.25 \mathrm{~mm}$

$\mu(\operatorname{MoK} \alpha)=12.47 \mathrm{~cm}^{-1}$

transmissn: 0.688 (max), $0.437(\min )$

no. of unique data: 2554

no. of data with I > 2 $\sigma(\mathrm{I}): 1911$

no. of atoms, params: 37,164

$\max \Delta / \sigma$ ratio: 0.0006

$\mathrm{R}_{\mathrm{F}} ; \mathrm{R}_{\mathrm{w}}$ : 0.028; 0.028

GOF: 1.82

D-map, $\max / \min : 0.60 /-0.74 \mathrm{e} / \AA^{3}$

Chart 1

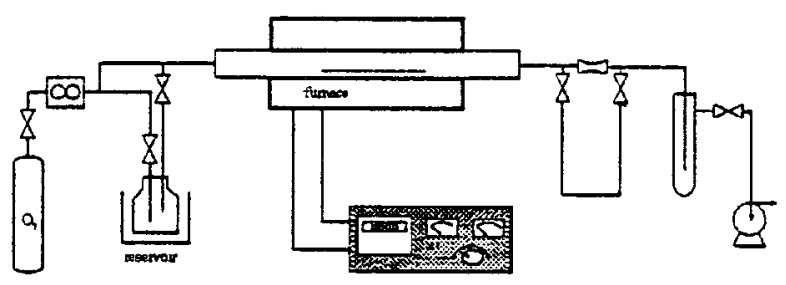

RCA cleaning method; $\mathrm{SiO}_{2}$ substrates were placed in dilute HF solution for $12 \mathrm{~h}$, and then washed with deionized water and dried at $120^{\circ} \mathrm{C}$ for $10 \mathrm{~min}$. The flow rate of carrier gas was adjusted to $20-40 \mathrm{~mL} / \mathrm{min}$. The duration of deposition was typically between $30 \mathrm{~min}$ and $1 \mathrm{~h}$.

\section{Results and Discussion}

Synthesis and Characterization of Precursors. The synthesis of $\beta$-ketoi mineligands involved the simple condensation of acetylacetone and a primary amine $\left(\mathrm{H}_{2^{-}}\right.$ $\mathrm{NR}^{1} ; \mathrm{R}^{1}=\mathrm{Ph}, \mathrm{Me}, \mathrm{Bu}^{\mathrm{n}}$, methoxyethyl, allyl) in an aromatic solvent. ${ }^{6} \mathrm{After}$ the mixture was stirred at room temperature for 2 days, the free $\beta$-ketoimine was then isolated in $75-89 \%$ yields. The preparations involving hexafluoroacetylacetone (hfacH) utilized K-10 montmorillonite as a catalyst ${ }^{7}$ to afford the analogous fluorine-substituted $\beta$-ketoimine ligand in $60-85 \%$ yield upon heating in $\mathrm{CHCl}_{3}$ solvent for 2 days (Scheme 1 ). Formation of the ammonium salt $[\mathrm{hfac}]\left[\mathrm{NH}_{3} \mathrm{R}\right],{ }^{8}$ a kinetic product obtained by direct mixing of hexafluoroacetylacetone with amine, was not observed. The latter synthetic procedure is effective for the large-scale generation of the required bis(trifluoromethyl)-substituted $\beta$-ketoimine ligands.

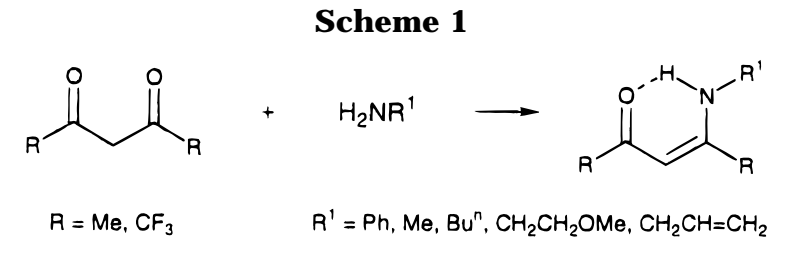


Table 2. Structures and Melting Points of Palladium Precursors 1-16

\begin{tabular}{|c|c|c|c|c|c|c|c|}
\hline compd no. & $\mathrm{R}$ & $\mathrm{R}^{1}$ & $R^{2}$ & $\mathrm{R}^{3}$ & $\mathrm{R}^{4}$ & yield (\%) & $\mathrm{mp}\left({ }^{\circ} \mathrm{C}\right)$ \\
\hline 1 & $\mathrm{Me}$ & $\mathrm{Ph}$ & $\mathrm{Me}$ & $\mathrm{H}$ & $\mathrm{H}$ & 86 & $129-132$ \\
\hline 2 & $\mathrm{Me}$ & $\mathrm{Me}$ & $\mathrm{H}$ & $\mathrm{H}$ & $\mathrm{H}$ & 61 & unstable \\
\hline 3 & $\mathrm{Me}$ & $\mathrm{Me}$ & $\mathrm{Me}$ & $\mathrm{H}$ & $\mathrm{H}$ & 80 & $79-81^{a}$ \\
\hline 4 & $\mathrm{Me}$ & $\mathrm{Me}$ & $\mathrm{H}$ & $\mathrm{Me}$ & $\mathrm{H}$ & 86 & $89-91^{a}$ \\
\hline 5 & $\mathrm{Me}$ & $\mathrm{CH}_{2} \mathrm{CH}_{2} \mathrm{OMe}$ & $\mathrm{H}$ & $\mathrm{Me}$ & $\mathrm{H}$ & 90 & $49-51^{a}$ \\
\hline 6 & $\mathrm{Me}$ & $\mathrm{CH}_{2} \mathrm{CH}=\mathrm{CH}_{2}$ & $\mathrm{H}$ & $\mathrm{Me}$ & $\mathrm{H}$ & 80 & liquid \\
\hline 7 & $\mathrm{Me}$ & $\mathrm{Me}$ & $\mathrm{H}$ & $\mathrm{Me}$ & $\mathrm{Me}$ & 90 & $70-72^{a}$ \\
\hline $8^{\mathrm{b}}$ & $\mathrm{Me}$ & $\mathrm{CH}_{2} \mathrm{CH}_{2} \mathrm{OMe}$ & $\mathrm{H}$ & $\mathrm{Me}$ & $\mathrm{Me}$ & 87 & liquid \\
\hline $9^{b}$ & $\mathrm{Me}$ & $\mathrm{CH}_{2} \mathrm{CH}_{2} \mathrm{OMe}$ & $\mathrm{Me}$ & $\mathrm{CH}_{2} \mathrm{OMe}$ & $\mathrm{Me}$ & 73 & liquid \\
\hline $10^{b}$ & $\mathrm{CF}_{3}$ & $\mathrm{Me}$ & $\mathrm{Me}$ & $\mathrm{CH}_{2} \mathrm{OMe}$ & $\mathrm{Me}$ & 90 & liquid \\
\hline $11^{b}$ & $\mathrm{CF}_{3}$ & $\mathrm{CH}_{2} \mathrm{CH}_{2} \mathrm{OMe}$ & $\mathrm{Me}$ & $\mathrm{CH}_{2} \mathrm{OMe}$ & $\mathrm{Me}$ & 75 & $79-81$ \\
\hline 12 & $\mathrm{CF}_{3}$ & $\mathrm{Me}$ & $\mathrm{H}$ & $\mathrm{H}$ & $\mathrm{H}$ & 95 & $118-120$ \\
\hline 13 & $\mathrm{CF}_{3}$ & $B u^{n}$ & $\mathrm{H}$ & $\mathrm{H}$ & $\mathrm{H}$ & 87 & $60-62$ \\
\hline 14 & $\mathrm{CF}_{3}$ & $\mathrm{CH}_{2} \mathrm{CH}_{2} \mathrm{OMe}$ & $\mathrm{H}$ & $\mathrm{H}$ & $\mathrm{H}$ & 88 & $71-73$ \\
\hline 15 & $\mathrm{CF}_{3}$ & $\mathrm{Me}$ & $\mathrm{H}$ & $\mathrm{Me}$ & $\mathrm{H}$ & 95 & $87-89$ \\
\hline 16 & $\mathrm{CF}_{3}$ & $\mathrm{CH}_{2} \mathrm{CH}=\mathrm{CH}_{2}$ & $\mathrm{H}$ & $\mathrm{Me}$ & $\mathrm{H}$ & 80 & $89-91$ \\
\hline
\end{tabular}

a Sample decomposed upon melting. ${ }^{\text {b }}$ Selected for CVD experiments.

Scheme 2

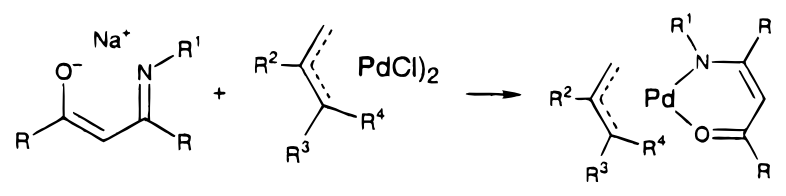

The complexes $[(\text { allyl }) \mathrm{Pd}(\mu-\mathrm{Cl})]_{2}$ were prepared according to literature methods. ${ }^{5}$ Five allyl complexes were synthesized; their molecular structures are<smiles></smiles>

$R^{2}, R^{3}, R^{4}=H, H, H ; M e, H, H ; H, M e, H$

$\mathrm{Me}, \mathrm{Me}, \mathrm{Me} ; \mathrm{Me}, \mathrm{CH}_{2} \mathrm{OMe}, \mathrm{Me}$

Our synthetic strategy was to select congested, highly substituted allyl ligands while avoiding unnecessarily large organic substituents which could reduce the volatility of the palladium complexes.

In the synthesis of allyl( $\beta$-ketoiminato)palladium(II) complexes, the $\beta$-ketoimine ligand is first converted to the $\beta$-ketoiminate salt on treatment with a slight excess of $\mathrm{NaOH}$ in aqueous media or $\mathrm{NaOMe}$ in diethyl ether. Mixing this solution with a second solution containing $[(\text { allyl }) \mathrm{Pd}(u-\mathrm{Cl})]_{2}$ in equal proportion generates the allyl $\beta$-ketoiminato compl exes (allyl $=\mathrm{C}_{3} \mathrm{H}_{5}, \mathrm{C}_{3} \mathrm{H}_{4} \mathrm{Me}, \mathrm{C}_{3} \mathrm{H}_{3}-$ $\mathrm{Me}_{2}, \mathrm{C}_{3} \mathrm{H}_{2} \mathrm{Me}_{2}\left(\mathrm{CH}_{2} \mathrm{OMe}\right.$ ); Scheme 2). The $\beta$-ketoi minato $\mathrm{Pd}$ complexes prepared during this study are listed in Table 2, al ong with their structural properties, reaction yields, and melting points.

The physical properties of the $\beta$-ketoiminate complexes differ substantially: 1 possesses the highest melting point and is relatively nonvolatile at room temperature, while $\mathbf{2}$ is unstable at room temperature and even decomposes to form a Pd film on the wall of the reaction flask during workup. Complexes 1, 8-10, and 14-16 can be stored at room temperature for long periods, whereas other less stable anal ogues have to be kept bel ow $0{ }^{\circ} \mathrm{C}$ to avoid decomposition. The thermal stability of the Pd complexes can be improved by

(8) Shin, H.-K.; Hampden-Smith, M.J .; Kodas, T. T.; Rheingold, A. L. J . Chem. Soc., Chem. Commun. 1992, 217. changing the substituents $R$ on the ketoimine ligand from $\mathrm{Me}$ to $\mathrm{CF}_{3}$, increasing the numbers of methyl substituents on the $\eta^{3}$-allyl ligand, or attaching a bulky or long-chain hydrocarbon, such as an n-butyl, methoxyethyl, or allyl group to the nitrogen of the ketoiminato ligand. Evidently, steric interactions on both the $\eta^{3}$-allyl and $\beta$-ketoiminateligands is the principal factor that stabilizes these complexes. The presence of a methoxy and a second allyl pendant also seems to improve the thermal stability.

The volatility and thermal stability of the Pd compounds were investigated, since these are important in CVD applications. Using thermogravimetric analysis (TGA) it was found that Pd complexes with ketoimine ligands derived from acetylacetone are less thermally stable. This effect is best seen from the TGA of $\mathbf{8}$, which exhibits a sudden weight loss of $68 \%$ between 146 and $182{ }^{\circ} \mathrm{C}$ and which leaves a black residue nearly equivalent to the theoretical weight of Pd metal. In contrast, complexes derived from hexafluoroacetylacetone are more volatile and robust under conditions of TGA experiments. Thus, most of these fluorine-substituted complexes partially evaporated and partially decomposed, producing a small amount of metallic Pd residue. Of particular interest is the TGA of $\mathbf{1 0}$, which showed a simple one-step loss of nearly $100 \%$ within the temperature range $139-175{ }^{\circ} \mathrm{C}$. This observation is consistent with highly effective transportation of $\mathrm{Pd}$ metal into the gas phase without decomposition.

The physical properties, such as the melting point, of the $\mathrm{Pd}$ complexes can be modified by changing the ligand environment to give liquid CVD precursors having distinct advantages in applications due to their reproducible and steady rates of evaporation. ${ }^{9}$ As indicated in Table 2, complexes 4-12 possess fairly low melting points and show potential for CVD application as liquid precursors, while complexes 8-10 exist as slightly viscous liquid materials at room temperature.

Structure of Ketoi mi nato Complexes. Confirmation of the molecular structure was provided by a singlecrystal X-ray analysis of the N-substituted phenyl complex 1 . As can be seen from Figure 1, the Pd atom possesses a distorted-square-planar coordination environment consisting of $\mathrm{O}$ and $\mathrm{N}$ atoms of the $\beta$-ketoiminate ligand and $C(1)$ and $C(3)$ of the allyl ligand. The $\mathrm{Pd}-\mathrm{O}$ distance $(2.056(3) \AA)$ is nearly equal to that of

(9) (a) Maury, F. J . Phys. IV 1995, C5, 449. (b) Maury, F. Chem. Vap. Deposition 1996, 2, 113. 


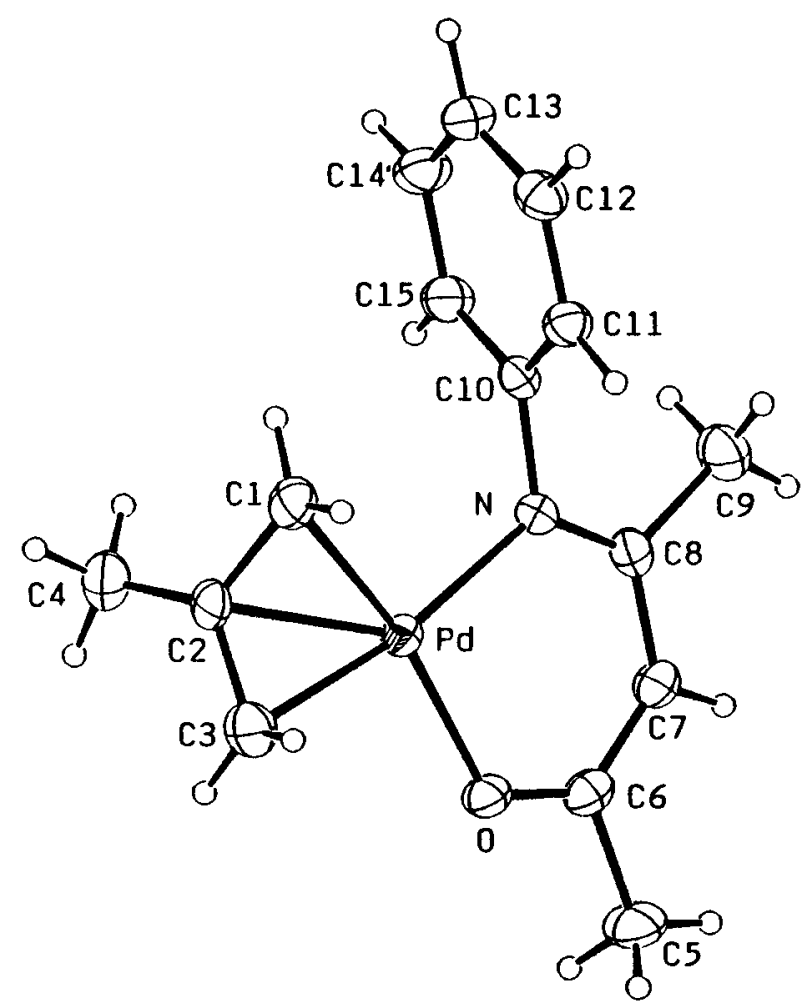

Figure 1. Molecular structure of complex 1, emphasizing the coordination environment about $\mathrm{Pd}$ and showing the atomic numbering scheme. Relevant bond distances $(\AA)$ : $\mathrm{Pd}-\mathrm{O}=2.056(3), \mathrm{Pd}-\mathrm{N}=2.066(3), \mathrm{N}-\mathrm{C}(8)=1.308(5)$, $\mathrm{O}-\mathrm{C}(6)=1.275(5), \mathrm{C}(6)-\mathrm{C}(7)=1.372(6), \mathrm{C}(7)-\mathrm{C}(8)=$ 1.407(6), $\mathrm{Pd}-\mathrm{C}(1)=2.097(4), \mathrm{Pd}-\mathrm{C}(2)=2.116(4), \mathrm{Pd}-\mathrm{C}(3)$ $=2.139(4), \mathrm{C}(1)-\mathrm{C}(2)=1.410(6), \mathrm{C}(2)-\mathrm{C}(3)=1.399(6)$. Bond angles (deg): $\mathrm{O}-\mathrm{Pd}-\mathrm{N}=91.2(1), \mathrm{N}-\mathrm{Pd}-\mathrm{C}(1)=$ 101.5(2), O-Pd-C(3) = 99.3(2).

the $\mathrm{Pd}-\mathrm{N}$ distance $(2.066(3) \AA)$, while the $\mathrm{N}-\mathrm{C}(8)$ distance $(1.308(5) \AA$ ) and its adjacent $C(7)-C(8)$ distance $(1.407(6) \AA)$ are also similar to the $\mathrm{O}-\mathrm{C}(6)$ distance $(1.275(5) \AA)$ and the respective $C(6)-C(7)$ distance (1.372(6) $\AA$ ), indicating formation of evenly distributed and del ocalized $\pi$-interactions on the ketoiminato ligand. The $\mathrm{Pd}-\mathrm{C}$ distances and the $\mathrm{N}-\mathrm{Pd}-\mathrm{C}(1)$ and $\mathrm{O}-\mathrm{Pd}-$ $C(3)$ angles, respectively, are within the limits of experimental error of the related ketoi minate complex, but the latter contains a nondelocalized ketoiminato ligand. ${ }^{10}$

For compounds that contain an asymmetric $\eta^{3}$-allyl ligand, only one set of ${ }^{1} \mathrm{H}$ NMR proton signals were observed for all examples at even lower temperature. Thus, only one isomer is present in solution for the asymmetric $\eta^{3}$-allyl complexes, in which the nonhydrogen substituents are believed to be located at the position trans to the nitrogen atom of the $\beta$-ketoiminate ligand, because of reduced steric interaction.

Fluxional Behavior in Solution. The allyl $(\beta-k e-$ toiminato)palladium complexes possess novel dynamic behavior in solution, as revealed from the variabletemperature ${ }^{1} \mathrm{H}$ NMR studies of methallyl complexes $3,6,15$, and 16 in $\mathrm{CDCl}_{3}$, toluene, or DMSO. For instance, olefinic proton signals of the methallyl ligand in complex 6 appear at $\delta 3.48(\mathrm{~J} \mathrm{Hн}=2.8 \mathrm{~Hz}), 2.72,2.58$ $(\mathrm{J} \mathrm{HH}=2.8 \mathrm{~Hz})$, and $2.33 \mathrm{in} \mathrm{d}_{8}$-toluene at room temper-

(10) Claverini, R.; Ganis, P.; Pedone, C. J . Organomet. Chem. 1973, $50,327$.

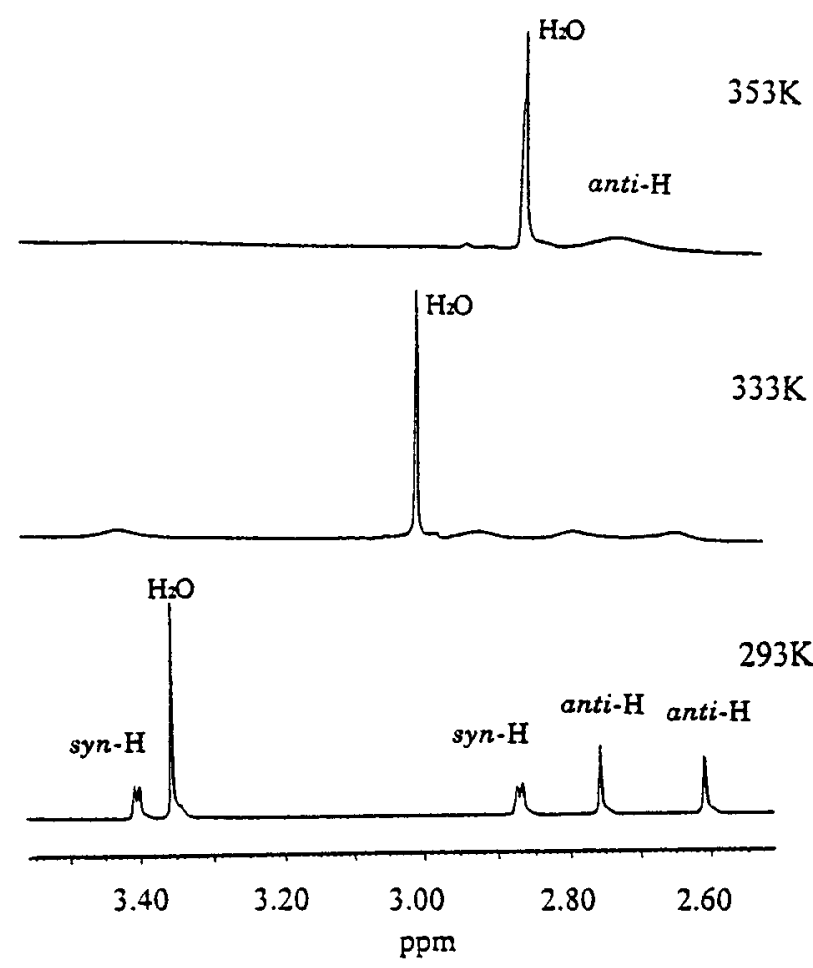

Figure 2. Variable-temperature ${ }^{13} \mathrm{C}$ NMR spectra $\left(d_{6}-\right.$ DMSO) of $\mathbf{6}$, showing the ol efinic protons of the methallyl ligand.

ature. These four signals are assigned to syn, anti, syn, and anti protons on the basis of the pattern of $4 \mathrm{JH}$ coupling observed. When the temperature is raised to $343 \mathrm{~K}$, all signals broadened substantially, indicating the onset of chemical exchange. This behavior was confirmed by a ${ }^{1} \mathrm{H}$ NMR spin saturation transfer experiment conducted on complex 3 in $\mathrm{CDCl}_{3}$ solution at room temperature. In the latter experiment, irradiation of one anti proton signal led to the complete suppression of the second anti signal but gave no obvious diminution of the syn proton signals. The same observation was noted on irradiation of the syn proton signals under similar conditions. These ${ }^{1} \mathrm{H}$ NMR SST experiments clearly indicate the presence of a dynamic process that causes the pairwise exchange between pairs of anti protons and syn protons but produces no scrambling among the anti and syn protons.

The fluxional behavior was observed to be more rapid in a polar organic solvent. This is best exemplified by the variable-temperature ${ }^{1} \mathrm{H}$ NMR studies of complex 6 in $d_{6}$-DMSO. As seen in Figure 2 , the syn protons at $\delta 3.41$ and 2.87 and the anti protons at $\delta 2.75$ and 2.61 observed at room temperature broaden substantially when the temperature is increased. When the temperature is raised to $353 \mathrm{~K}$, the anti protons produce a broad signal at $\delta 2.71$, while the syn protons completely merge into the baseline; no coalescence of syn protons was observed at this temperature because their chemical shifts differ greatly. Moreover, the ${ }^{1} \mathrm{H}$ NMR spectrum of $\mathbf{1 6}$ in $\mathrm{d}_{6}$-DMSO at room temperature exhibits only two signals: one is a broad signal at $\delta 3.62$ and the second is a relatively sharp signal at $\delta 3.07$ due to syn and anti protons, which is in marked contrast to observation of four distinct ol efinic signals of $\eta^{3}$-allyl ligand in $\mathrm{CDCl}_{3}$ or $\mathrm{d}_{8}$-toluene solution, confirming the 
Scheme 3<smiles>[R]C(=O)O[R8]([H])N([R])C([R])=CC(=C)C</smiles>

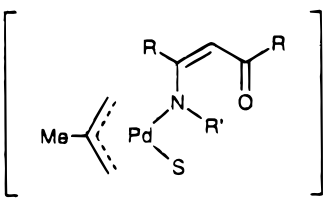

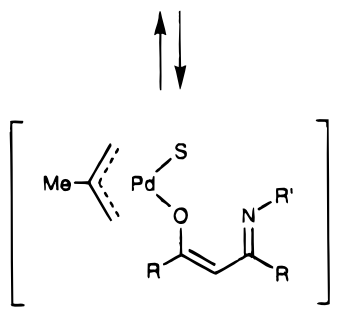<smiles>C=C(C)CN(P)N(P)C(=O)C=CC</smiles><smiles>[V][V]</smiles>

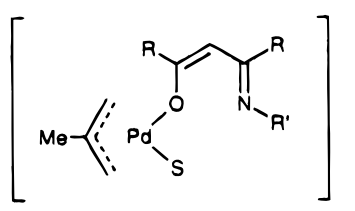<smiles>C=C=C</smiles><smiles>[R]ON([P])/C([R])=C\C([R])=C</smiles>

existence of a similar, but much faster, pairwise exchange of syn and anti protons.

The lack of interchange between syn and anti protons in all experiments clearly shows that the fluxional process is not due to a $\eta^{3}-\eta^{1}-\eta^{3}$ interconversion of the allyl ligand. ${ }^{11}$ Other likely explanations would be $180^{\circ}$ rotation of the planar $\eta^{3}$-allyl ligand about the allylpalladium axis ${ }^{12}$ and the formation of a solventcoordinated intermediate that contains either an $\mathrm{O}$ bound or a N-bound monodentate ketoiminato ligand, followed by interchange of the solvent molecule and the ketoiminato ligand (Scheme 3), as both latter processes would average the environment of syn-syn and antianti protons, but not interchange syn and anti protons. Direct rotation of the allyl ligand seems to be the process occurring in chloroform or toluene, as these nonpolar solvents coordinate poorly to the Pd atom. However, on altering the solvent to DMSO, incorporation of the solvent molecule into the coordination sphere of the palladium atom is more facile; thus, the solvent-assisted pathway becomes the dominant exchange process in solution. Interestingly, this exchange pathway is mechanistically similar to that observed in palladium complexes [(allyl)PdCIL], generated by the addition of DMSO, phosphine, or arsine ligand, to allylpalladium chloride complexes [(allyl)Pd( $u-\mathrm{Cl})]_{2} \cdot{ }^{13}$

CVD of Palladium. CVD experiments were conducted in a horizontal hot wall reactor with selected ketoiminato precursors $\mathbf{8}-\mathbf{1 1}$ at reduced pressure. Other complexes were not tested in CVD experiments because of Iow volatility and poor thermal stability. The experiments were carried out at $200-300{ }^{\circ} \mathrm{C}$ and precursor samples were kept between 25 and $70{ }^{\circ} \mathrm{C}$, with oxygen as carrier gas (20-40 $\mathrm{mL} / \mathrm{min}$ ) to suppress carbon

(11) (a) Cotton, F. A.; Faller, J . W.; Musco, A. I norg. Chem. 1967, 6, 179. (b) van Leeuwen, P. W. N. M.; Praat, A. P. J . Chem. Soc., Chem. Commun. 1970, 365.

(12) (a) Davison, A.; Rode, W. C. Inorg. Chem. 1967, 6, 2124. (b) Faller, J . W.; I ncorvia, M. J . Inorg. Chem. 1968, 7, 840. (c) Faller, J. W.; Incorvia, M. J .; Thomsen, M. E. J . Am. Chem. Soc. 1969, 91, 518.

(13) Ramey, K. C.; Statton, G. L. J . Am. Chem. Soc. 1966, 88, 4387.
Table 3. Deposition Conditions and Thin-Film Properties $^{\mathrm{a}}$

\begin{tabular}{ccccccc}
\hline & sample & deposition & \multirow{2}{*}{$\mathrm{O}_{2}$ flow } & \multicolumn{3}{c}{ atom $\%$} \\
\cline { 5 - 7 } precursor & temp $\left({ }^{\circ} \mathrm{C}\right)$ & temp $\left({ }^{\circ} \mathrm{C}\right)$ & rate $(\mathrm{mL} / \mathrm{min})$ & $\mathrm{Pd}$ & $\mathrm{C}$ & $\mathrm{F}$ \\
\hline $\mathbf{8}$ & 70 & 300 & 10.0 & 95 & 4.2 & \\
$\mathbf{8}$ & 70 & 300 & 40.0 & 98 & 1.5 & \\
$\mathbf{8}$ & 70 & 200 & 40.0 & 98 & 2.0 & \\
$\mathbf{9}$ & 70 & 250 & 40.0 & 98 & 1.2 & \\
$\mathbf{1 0}$ & 25 & 200 & 34.0 & 97 & 1.5 & 1.4 \\
$\mathbf{1 0}$ & 60 & 200 & 25.0 & 97 & 1.4 & 1.6 \\
$\mathbf{1 1}$ & 60 & 300 & 35.0 & 97 & 1.2 & 1.2 \\
$\mathbf{1 1}$ & 60 & 200 & 25.0 & 97 & 1.4 & 2.1
\end{tabular}

a All experiments were carried out under a pressure of $(1-3) \times$ $10^{-2}$ Torr, depending on the $\mathrm{O}_{2}$ flow rate; substrates were broken Si wafers.

impurities. ${ }^{14}$ Although experimental conditions were not optimized, the Pd films obtained were shiny and mirrorlike. They adhered well to silicon substrates but poorly to $\mathrm{SiO}_{2}$, as tested by scratch or adhesive-tape tests. Preliminary XPS and Auger spectroscopy identify the films prepared from $\mathbf{8}$ and $\mathbf{9}$ as $\geq 98 \%$ Pd with $\leq 2 \%$ of carbon and no nitrogen contamination, while films from $\mathbf{1 0}$ and $\mathbf{1 1}$ differ in that detectable amounts $(<2 \%)$ of residual fluorine are retained, when the deposition experiments were conducted at $200-300^{\circ} \mathrm{C}$. However, when the deposition temperature is increased to $300^{\circ} \mathrm{C}$ and the flow rate of oxygen carrier gas to $40 \mathrm{~mL} / \mathrm{min}$, the amount of carbon impurities dropped to $\sim 1 \%$, which represents the best film we obtained in this work. A summary of typical experimental conditions, together with analytical data for palladium films, is given in Table 3. Electrical conductivity measurement of films in one example give a resistivity of $1.6 \times 10^{-5} \Omega \mathrm{cm}$, slightly higher than that of bulk Pd metal $\left(1.1 \times 10^{-5}\right.$ $\Omega \mathrm{cm}$ ). Again, this effect is attributed to incorporation of carbon impurities in the Pd films.

Conclusion. Allyl ( $\beta$-ketoiminato)pal ladium(II) complexes can serve as precursors for chemical vapor deposition of Pd thin films. Unlike diketonato compounds reported previously, ${ }_{4}$ which require fairly expensive fluorinated diketonato ligands, the compounds discussed herein can be prepared in good yields from easily accessible and inexpensive commercially available materials. F urthermore, the melting points and thermal stabilities of these precursors can be tailored by systematic modification of the $\eta^{3}$-allyl or $\beta$-ketoiminato group. After appropriate adjustment of substituents, some compl exes display excellent thermal stability and volatility at or above room temperature. In the case of complexes 8-10, stable liquid phases are obtained: they are among the best CVD precursors for Pd thin films reported to date.

Acknowledgment. We thank the National Science Council of the Republic of China for financial support (Grant No. NSC 87-2113-M007-027-COM).

Supporting Information Available: Tables of atomic coordinates and anisotropic thermal parameters for complex $\mathbf{1}$ and ${ }^{1} \mathrm{H}$ NMR spectra for complexes $\mathbf{2}, \mathbf{6}, \mathbf{8}-\mathbf{1 1}, \mathbf{1 3}$, and 14. This material is available free of charge via the Internet at http://pubs.acs.org.

\section{OM980728C}

(14) Hierso, J .-C.; Satto, C.; Feurer, R.; Kalck, P. Chem. Mater. 1996, 8,2481 . 